\title{
Bevacizumab Use for Recurrent High- Grade Glioma at McGill University Hospital
}

\author{
Solmaz Sahebjam, Evgenia Garoufalis, Marie-Christine Guiot, Thierry Muanza, \\ Rolando Del Maestro, Kevin Petrecca, Rajesh Sharma, Petr Kavan
}

\begin{abstract}
Background: Bevacizumab, a humanized recombinant anti-vascular endothelial growth factor antibody, was approved in Canada in 2010 for the treatment of high-grade glioma. We report the effectiveness and safety of bevacizumab in the treatment of patients with recurrent high-grade gliomas at a single institution. Methods: Twenty-seven consecutive patients with high-grade glioma (anaplastic glioma and glioblastoma) at first or subsequent relapse were treated with bevacizumab alone or in combination with chemotherapy. The primary endpoint was progression-free survival (PFS) and secondary endpoints were objective response rate, six month PFS, overall survival (OS), and safety profile. Results: The clinical benefit rate (complete and partial responses plus stable disease) was 59\%. Median PFS was 4.3 (95\% CI, 3.0-10.9) months, with a six month PFS rate of $43 \%$. Median OS after current relapse was 8.9 (95\% CI, 5.8-not reached) months. Ten episodes of grade $3 / 4$ adverse events were observed in nine patients, including fatigue $(\mathrm{n}=3)$, thrombocytopenia $(\mathrm{n}=4)$, and myelotoxicity, febrile neutropenia, and pulmonary embolism $($ each $\mathrm{n}=1)$. Conclusions: We consider the efficacy and safety profile of bevacizumab is comparable to other cohorts of patients treated for recurrent high-grade glioma at other international institutions.
\end{abstract}

RÉSUMÉ: L'utilisation du Bevacizumab dans le traitement de la récidive d'un gliome de haut grade de malignité au Centre universitaire de santé McGill. Contexte : Le Bevacizumab, un anticorps recombinant humanisé dirigé contre le facteur de croissance endothélial vasculaire, a été approuvé au Canada en 2010 pour le traitement du gliome de haut grade de malignité. Nous rapportons l'efficacité et la sécurité du Bevacizumab dans le traitement de patients présentant une récidive de gliomes de haut grade de malignité dans notre institution. Méthode : Vingt-sept patients consécutifs atteints de gliomes de haut grade de malignité (gliomes anaplasiques et glioblastomes) ont été traités par le Bevacizumab seul ou en combinaison à la chimiothérapie à la première récidive ou au moment de récidives subséquentes. Le critère d'évaluation primaire était la survie sans progression (SSP) et les critères d'évaluation secondaires étaient le taux de réponse objective, une SSP de 6 mois, la survie globale (SG), et le profil de sécurité. Résultats : Le taux de bénéfice clinique (réponse complète et partielle avec maladie stable) était de 59\%. La SSP médiane était de 4,3 mois (IC à 95\% : 3,0 à 10,9) avec un taux de SSP de 6 mois de 43\%. La SG médiane après la récidive en cours était de 8,9 mois (IC à 95\% de 5,8 à non atteinte). Dix épisodes d'incidents thérapeutiques de grade $3 / 4$ ont été observés chez 9 patients, dont de la fatigue $(n=3)$, une thrombocytopénie $(n=4)$ et une myélotoxicité, une neutropénie fébrile et une embolie pulmonaire $(\mathrm{n}=1$ chacun). Conclusions : Nous considérons que le profil d'efficacité et de sécurité du Bevacizumab que nous avons observé est comparable à celui d'autres cohortes de patients traités pour une récidive d'un gliome de haut grade de malignité dans d'autres institutions internationales.

Can J Neurol Sci. 2013; 40: 241-246

High-grade gliomas are the most common and aggressive group of primary central nervous system (CNS) tumors in adults and are characterized by diffuse infiltration of brain parenchyma and prominent angiogenesis. Their treatment has always been problematic and, despite recent significant advances in brain imaging, neurosurgery, and medical therapy ${ }^{1}$, prognosis remains dismal. Glioblastoma is currently associated with a median overall survival (OS) of approximately 14.5 months after diagnosis and six months after recurrence ${ }^{2}$; median progressionfree survival is approximately 7.0 months after diagnosis. For anaplastic astrocytomas, median OS is two to three years after diagnosis and approximately 11 months after recurrence ${ }^{3}$.

New therapeutic targets have recently emerged following advances elucidating the pathogenesis of gliomas. One target that has emerged is vascular endothelial growth factor (VEGF), a key protein regulator of new blood vessel formation and tumor angiogenesis. Vascular endothelial growth factor overexpression is related to the degree of tumor development and prognosis ${ }^{4}$. In particular, glioblastoma is characterized by sustained angiogenesis and frequently exhibits VEGF overexpression ${ }^{5,6}$.

From the Drug Development Program (SS), Princess Margaret Hospital, Toronto, Ontario; Department of Oncology (EG, RS, PK), Department of Pathology (MCG), Division of Radiation Oncology (TM), Department of Oncology and Segal Cancer Centre, McGill University, Department of Neurology and Neurosurgery (RDM, KP), Montreal Neurological Institute and Hospital, McGill University, Montreal, Quebec; Canada.

Received June 20, 2012. Final Revisions Submitted September 19, 2012 Correspondence to: Petr Kavan, McGill University, Royal Victoria Hospital, Department of Oncology, 687 Pine Avenue West, Montreal, Quebec, H3A 1A1, Canada. Email: petr.kavan@mcgill.ca. 
Bevacizumab (Avastin ${ }^{\circledR}$ ) is a therapeutic humanized recombinant monoclonal antibody that binds to and inhibits the activity of $\mathrm{VEGF}^{7}$. It has shown activity against various tumors such as colorectal, lung, renal, ovarian, and breast cancer ${ }^{8-11}$. Preclinical models show that bevacizumab has activity against glioblastoma both alone and in combination with radiotherapy and temozolomide ${ }^{12,13}$. Several clinical studies have demonstrated the activity of bevacizumab in the treatment of recurrent gliomas when used as monotherapy or in combination with chemotherapy ${ }^{14-17}$. The BRAIN phase II study in glioblastoma patients after first or second relapse following radiotherapy plus temozolomide revealed compelling results in patients treated with bevacizumab alone $(n=85)$ or bevacizumab plus irinotecan $(\mathrm{n}=82)$, respectively: six month progression-free survival (PFS) rate (43\% vs. 50\%), objective response rate (ORR) (28\% vs. 38\%), and median OS (9.2 vs. 8.7 months $)^{18}$. Notably, primary endpoints including ORR rate and six month PFS rate were significantly $(\mathrm{P}<0.0001)$ higher than the rates of $5 \%$ and $15 \%$, respectively, expected with salvage chemotherapy according to external historical controls. ${ }^{18}$ Because of these promising results in a disease with few treatment options, the US Food and Drug Administration approved the use of bevacizumab for the treatment of recurrent glioblastoma in May 2009 on the basis of phase II trial results ${ }^{19}$. Bevacizumab was subsequently approved in March 2010 by Health Canada as a single agent for the treatment of glioblastoma following relapse or progression.

In this report, we evaluate the effectiveness, feasibility, and safety of using bevacizumab in the treatment of patients with recurrent high-grade gliomas at a single Canadian cancer center.

\section{METHODS}

All consecutive adult ( $\geq 18$ years) patients who received bevacizumab alone or in combination with chemotherapy for recurrent high-grade glioma (anaplastic glioma and glioblastoma) at the Montreal Neurological Institute (McGill University, Canada) were retrospectively identified and their charts reviewed. Patients initiated treatment between July 2008 and December 2010. The choice of bevacizumab therapy as a single agent or combination with chemotherapy, as well as the choice of concomitant chemotherapeutic agent(s), was at the discretion of the attending physician who was directly responsible for the care of each patient and was also based on the patient's insurance status. Bevacizumab 5-10 $\mathrm{mg} / \mathrm{kg}$ was administered intravenously every two weeks.

All patients underwent brain magnetic resonance imaging (MRI) at baseline (immediately prior to bevacizumab-based chemotherapy) and four to eight weeks later to assess response. Interpretable fluid-attenuated inversion recovery (FLAIR) and post-gadolinium T1-weighted sequences were required for each assessment. Response to therapy was evaluated using RANO criteria $^{20}$ : complete response (CR) was defined as complete disappearance of all measurable disease; partial response (PR) as a $\geq 50 \%$ decrease in the largest cross-sectional tumor area; and progressive disease (PD) as a $\geq 25 \%$ increase in largest crosssectional tumor area or appearance of new lesion; all other conditions were considered stable disease (SD). Stability or improvement of FLAIR sequences was required to define CR, $\mathrm{PR}$, or SD. Distant recurrence (new enhancing foci distant from the original area of the enhancing tumor) and diffuse recurrence ( $\geq 25 \%$ increase in area of abnormal FLAIR hyperintensity) were also identified.

The primary endpoint of the analysis was PFS. Secondary endpoints were ORR, OS, six month PFS, and safety profile. Time-to-event data (OS and PFS) were analyzed using the Kaplan-Meier method. Overall survival was defined as the time from bevacizumab treatment initiation to death resulting from any cause and PFS as the time from bevacizumab treatment initiation to progression or death. Adverse events were graded according to NCI-CTCAE (v3.0) criteria. Information on adjunctive corticosteroid use was noted.

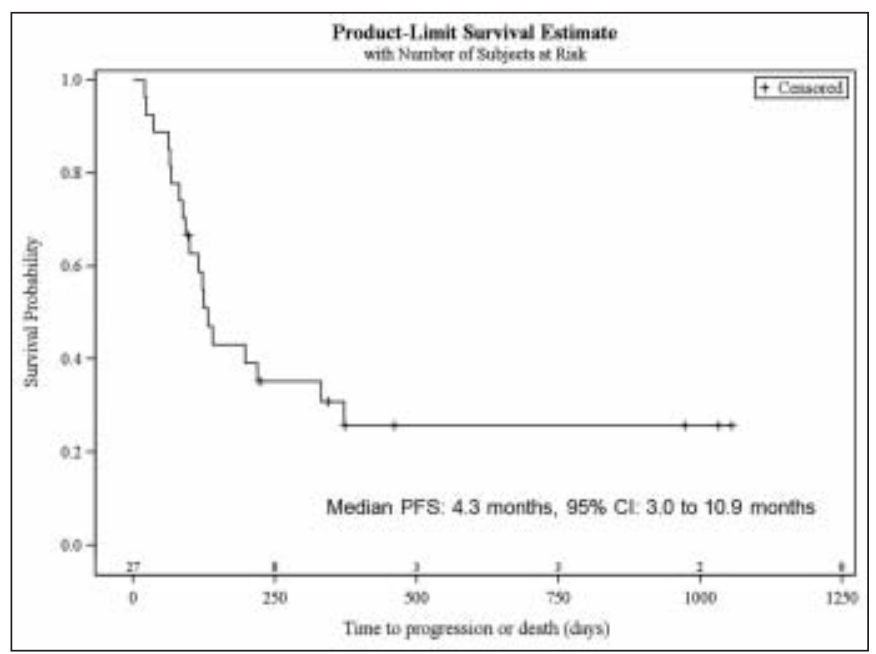

Figure 1: Kaplan-Meier plot for progression-free survival.

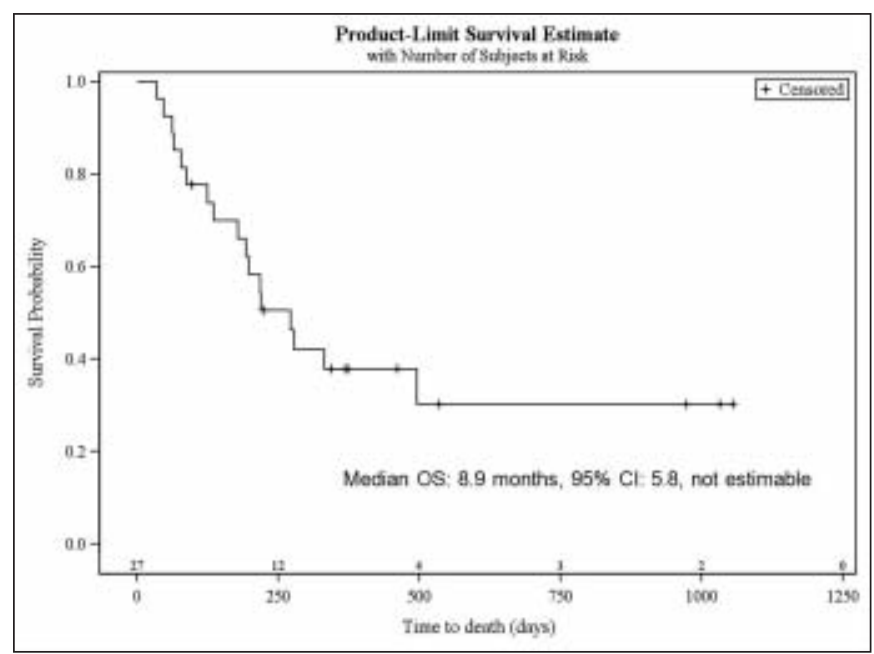

Figure 2: Kaplan-Meier plot for overall survival after relapse. 
Table 1: Characteristics of the research group

\begin{tabular}{|c|c|}
\hline \multicolumn{2}{|l|}{ Patient characteristics } \\
\hline $\begin{array}{l}\text { Patients, } n \\
\text { Median age at diagnosis, years (range) } \\
\text { Gender, } n(\%)\end{array}$ & $\begin{array}{c}27 \\
39(23-62)\end{array}$ \\
\hline Male & $15(56)$ \\
\hline Female & $12(44)$ \\
\hline \multicolumn{2}{|l|}{ Histologic diagnosis, $n(\%)$} \\
\hline Glioblastoma & $17(63)$ \\
\hline Anaplastic gliomas & $10(37)$ \\
\hline Median previous regimens, $n$ (range) & $2(0-4)$ \\
\hline \multicolumn{2}{|l|}{ Prior therapy, $n(\%)$} \\
\hline Surgery & $21(78)$ \\
\hline Gross total resection & 10 \\
\hline Subtotal/partial resection & 11 \\
\hline Radiotherapy & $24(89)$ \\
\hline Chemotherapy & $25(93)$ \\
\hline \multicolumn{2}{|l|}{ Prior chemotherapy, $n^{\star}$} \\
\hline Temozolomide monotherapy & 24 \\
\hline Temozolomide + procarbazine & 9 \\
\hline Temozolomide + everolimus & 1 \\
\hline Cabozantinib monotherapy & 1 \\
\hline Cediranib monotherapy & 1 \\
\hline Lomustine monotherapy & 1 \\
\hline Irinotecan monotherapy & 1 \\
\hline No chemotherapy & 2 \\
\hline \multicolumn{2}{|l|}{ Chemotherapy concurrent with bevacizumab, $n(\%)$} \\
\hline Temozolomide only & $4(15)$ \\
\hline Temozolomide + procarbazine & $11(41)$ \\
\hline Lomustine & $5(19)$ \\
\hline Irinotecan + temozolomide + procarbazine & $2(7)$ \\
\hline Monotherapy & $5(19)$ \\
\hline
\end{tabular}

*Indicates the number of patients who had received each specific chemotherapy at any time. Some of these patients may have received a specific chemotherapy on multiple occasions for different relapses.

\section{Results}

\section{Patient Characteristics}

Outcomes for a total of 27 consecutive patients treated with bevacizumab were reviewed. Baseline demographic and clinical characteristics of this study population are summarized in Table 1.

Histologic diagnosis was glioblastoma $(n=17)$, or anaplastic gliomas $(n=10)$. All patients had received prior surgery $(n=21)$ and/or radiotherapy $(n=24)$. Ten patients had received gross total resection and 11 subtotal/partial resection, while the remaining six patients had biopsy only. Twenty-five patients had received prior chemotherapy either first-line or following subsequent relapse/recurrence. Chemotherapy included temozolomide alone $(\mathrm{n}=24)$ or in combination with either procarbazine $(n=9)$ or everolimus $(n=1)$ and, in isolated cases, single-agent cabozantinib, cediranib, lomustine, and irinotecan. Cabozantinib and cediranib were given as part of clinical trials. The bevacizumab dose used was $5 \mathrm{mg}(\mathrm{n}=21), 7.5 \mathrm{mg}(\mathrm{n}=1)$ or $10 \mathrm{mg}(\mathrm{n}=5)$ every two weeks. Bevacizumab was most commonly used in combination or sequentially with chemotherapy (22 of 27 patients), including temozolomide with procarbazine $(\mathrm{n}=11)$, lomustine $(\mathrm{n}=5)$, temozolomide only ( $\mathrm{n}$ $=4)$, and temozolomide with procarbazine and irinotecan $(\mathrm{n}=$ 2). The remaining five patients received bevacizumab monotherapy. In addition, six patients received bevacizumab across multiple lines of treatment post-progression.

\section{Efficacy}

Best response to bevacizumab was as follows: PR $(n=1), S D$ $(n=15)$, PD $(n=9)$, and unknown $(n=2)$. For the overall population, ORR $(\mathrm{CR}+\mathrm{PR})$ was $3.7 \%$ and the clinical benefit rate $(\mathrm{CR}+\mathrm{PR}+\mathrm{SD})$ was $59 \%$. Median PFS was $4.3(95 \% \mathrm{CI}$, 3.0-10.9) months (Figure 1), with a six month PFS rate of $43 \%$. Median OS after current relapse was 8.9 (95\% CI, 5.8-not reached) months (Figure 2). There was no significant difference $(\mathrm{P}=0.66)$ in OS comparing the subpopulation who were treated with bevacizumab after first relapse $(n=9)$ and those treated after second or later relapse $(n=18$; some were treated up to sixth relapse) (Figure 3). There was a clinically significant reduction in cerebral edema in 12 patients $(44 \%)$. Steroid dose was decreased in 17 patients $(63 \%)$. 


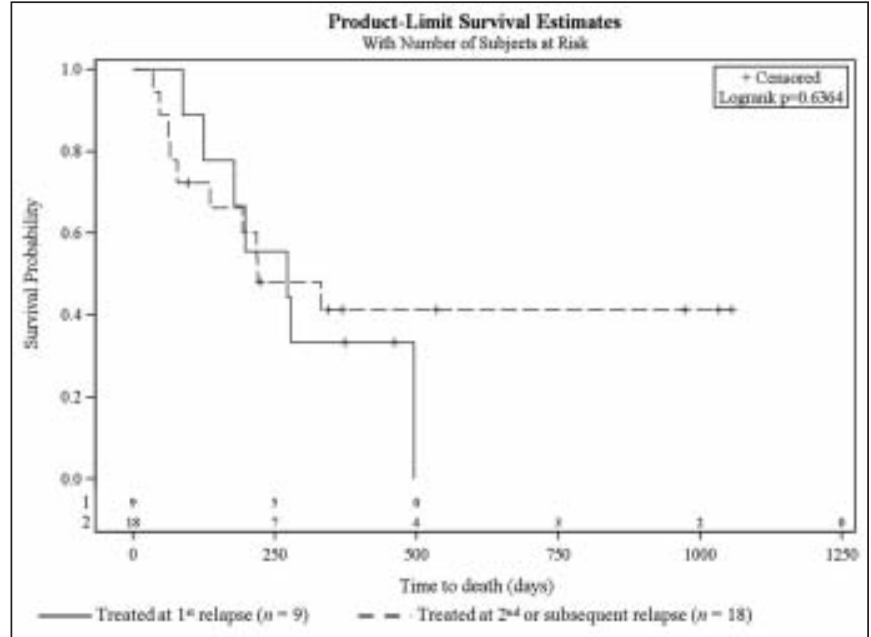

Figure 3: Kaplan-Meier plot for overall survival after bevacizumab treatment at first vs. second or subsequent relapse.

\section{Safety}

Ten episodes of grade 3/4 adverse events were observed in nine patients. These included grade 3 fatigue $(n=3)$; grade 3 thrombocytopenia $(n=2)$; grade 4 thrombocytopenia $(n=2)$; grade 4 myelotoxicity $(n=1)$; grade 4 pulmonary embolism ( $n$ $=1)$; and grade 3 febrile neutropenia $(\mathrm{n}=1)$.

\section{DISCUSSION}

Standard treatment for newly diagnosed glioblastoma includes surgery, followed by radiotherapy administered concomitantly with temozolomide, and then followed by adjuvant temozolomide treatment for 6-12 months ${ }^{21}$. Unfortunately, virtually all patients with high-grade glioma develop recurrent or progressive disease, usually within months after initial diagnosis. This is a particular problem in patients with glioblastoma. Therefore, alternative treatment strategies for patients at the time of disease progression need to be developed. The use of bevacizumab as an antiangiogenic agent in the treatment of high-grade glioma constitutes an important advance in the management of patients. Phase II studies conducted in United States and European centers have demonstrated a significant improvement in ORR and PFS in glioblastoma patients treated with bevacizumab and irinotecan ${ }^{14,16}$ when compared to conventional treatments ${ }^{22}$.

In our study we determined the efficacy and safety of bevacizumab in the treatment of patients with high-grade glioma (anaplastic gliomas and glioblastoma) at a single Canadian institution immediately after its approval in Canada. We were able to retrospectively evaluate bevacizumab in a cohort of 27 patients. We consider the efficacy of bevacizumab was favorable compared to other cohorts of patients with high-grade glioma treated with bevacizumab-based therapy for recurrent disease at other international institutions (Table 2). The clinical benefit rate $(\mathrm{CR}+\mathrm{PR}+\mathrm{SD})$ was $59 \%$. Median OS and PFS were 8.9 and 4.3 months, respectively, with a six month PFS rate of $43 \%$.

Table 2: Therapeutic outcome following treatment of recurrent high-grade glioma with bevacizumab plus chemotherapy or as monotherapy according to different studies

\begin{tabular}{|c|c|c|c|}
\hline Reference & Chemotherapy & No. of patients & Outcome \\
\hline \multirow[t]{2}{*}{ Current report } & Various $^{1}$ & 27 (17 grade IV; 10 grade III) & PFS6: 43\%, Median PFS: 4.3 mo \\
\hline & & & Median OS: 8.9 mo ORR: $3.7 \%$, CBR: $59 \%$ \\
\hline \multirow[t]{2}{*}{ Wong et al. ${ }^{23}$} & Various (meta-analysis) & 548 (all grade IV) & PFS6: $45 \%$, OS6: $76 \%$ \\
\hline & & & Median TTP: 6.1 months, ORR: $55 \%$ \\
\hline Poulsen et al. ${ }^{24}$ & Irinotecan & 52 (27 grade IV; 25 grade III) & PFS6: $32 \%$, ORR: $25 \%$ \\
\hline Soffietti et al. ${ }^{25}$ & Fotemustine & 31 (22 grade IV; 9 grade III) & Median TTP: 2.6 mo ORR: 35\% \\
\hline Reardon et al. ${ }^{26}$ & Etoposide & 59 (27 grade IV; 32 grade III) & PFS6: $44 \%$ in grade IV and $41 \%$ in grade III \\
\hline Guiu et al. $^{27}$ & Irinotecan & 77 (49 grade IV; 28 grade III) & ORR: $36 \%$ \\
\hline Gilbert et al. ${ }^{28}$ & Irinotecan & 57 (grade III/IV ${ }^{2}$ ) & PFS6: $37 \%$ \\
\hline \multirow[t]{3}{*}{ Sathornsumetee et al. ${ }^{29}$} & Erlotinib & 57 (25 grade IV; 32 grade III) & PFS6: $28 \%$ in grade IV and $44 \%$ in grade III \\
\hline & & & $\begin{array}{l}\text { Median OS: } 10.5 \text { mo in grade IV and } 17.5 \mathrm{mo} \text { in } \\
\text { grade III }\end{array}$ \\
\hline & & & ORR: $48 \%$ in grade IV and $31 \%$ in grade III \\
\hline \multirow[t]{2}{*}{ Verhoeff et al. ${ }^{30}$} & Dose-intense & 23 (15 grade IV; 8 grade III) & PFS6: $17.4 \%$, Median PFS: 3.5 mo \\
\hline & temozolomide & & Median OS: 4.4 mo ORR: $22 \%$ \\
\hline Elandt et al. ${ }^{31}$ & Liposomal doxorubicin & 21 (15 grade IV; 6 grade III) & PFS6: $19 \%$, Median PFS: 3.0 mo ORR: $48 \%$ \\
\hline \multirow[t]{2}{*}{ Calabrich et al. ${ }^{32}$} & Irinotecan or & 39 (27 grade IV; 10 grade III; 1 grade II) & Median PFS: 9.4 mo, Median OS: 18.7 mo \\
\hline & temozolomide & & PFS6: $61 \%$ \\
\hline Gallardo Martin et al. ${ }^{33}$ & Irinotecan & 15 (10 grade IV; 5 grade III) & PFS6: 73\%, Median PFS: 9.6 mo ORR: 53\% \\
\hline
\end{tabular}

${ }^{1}$ Two patients received monotherapy. ${ }^{2}$ Numbers for each grade not specified. Abbreviations: CBR, clinical benefit rate; mo, month; ORR, overall response rate; OS, overall survival; OS6, 6-month overall survival; PFS, progression-free survival; PFS6, 6-month progressionfree survival; TTP, time to progression. 
However, ORR at $3.7 \%$ was low compared to other studies. One potential reason for the low ORR was the assessment of response by FLAIR MRI instead of post contrast-infused MRI. Almost all responses were stable disease, which appears to reflect response to bevacizumab in other clinical indications. Median OS was comparable for patients treated with bevacizumab after first relapse compared to those after second or subsequent relapse, whereas median PFS and ORR were lower in the latter subpopulation (data not shown). It could therefore be speculated that earlier introduction of bevacizumab might translate into improved survival.

Direct comparison of these retrospective findings with those from other prospective reports is problematic and complicated by the following: 1) treated target populations differ with respect to the proportions of patients with different histologic grade, i.e. grade III versus grade IV disease, which is known to have a significant impact on prognosis; 2) the variety of chemotherapies used, which may potentially exhibit differential efficacy when used in combination with bevacizumab; and 3) the number of prior relapses experienced, with increasing number of relapses diminishing the likely response to therapy. The majority of our patients were treated with different combinations of classic chemotherapies, i.e. temozolomide \pm procarbazine $(56 \%)$, lomustine (19\%), and temozolomide + procarbazine + irinotecan $(8 \%)$, while five patients (19\%) received single-agent bevacizumab, and some had been treated intensively for up to six relapses. Despite evidence for bevacizumab + irinotecan being effective in the registration trial in glioblastoma, the majority of patients at our institution received a combination of bevacizumab with temozolomide and procarbazine; this decision was based on our previous experience with this regimen and because of concerns over toxicity related to irinotecan.

It is reasonable to expect that patients experiencing such a high number of prior relapses would be less likely to respond to further therapy. Nevertheless, median PFS, and the six month PFS rate in our cohort were generally at least or more favorable than those studies that included mixed populations with grade III and IV disease (Table 2). Table 2 does not include all studies of bevacizumab-based therapy in patients with a mixture of recurrent grade III/IV disease but does include most of the larger, recent studies in this setting. It does not include populations that were exclusively treated with bevacizumab-based therapy in the recurrent grade IV (glioblastoma) setting. Results in this exclusively grade IV situation are best summarized by the metaanalysis by Wong et $\mathrm{al}^{23}$, which is included in Table 2 for comparison. This meta-analysis included a total of 15 studies from 2005 to 2009, in which 548 patients (median age 53 years) with recurrent glioblastoma were treated with bevacizumabbased therapy. Median OS and six month PFS rate were 9.3 months and $45 \%$, respectively, which were comparable to our findings (8.9 months and 43\%).

Other clinical benefits associated with bevacizumab therapy included a reduction in steroid dose in a meaningful proportion of patients. Other reports have suggested important steroid dose reduction ${ }^{34,35}$. The safety profile of bevacizumab has already been well-established, notably in the BRAIN study ${ }^{18}$. No unexpected toxicities were observed in our series.

In conclusion, our findings in this small retrospective series of patients further support previously reported data that bevacizumab-based regimens are active in the treatment of patients with relapsed high-grade gliomas.

\section{ACKNOWLEDGMENTS}

Support for this project was provided by an educational grant from Roche Canada. Support for third-party writing assistance for this manuscript, provided by Operatrix Consulting Inc., was provided by Roche Canada.

\section{REFERENCES}

1. Adamson C, Kanu OO, Mehta AI, et al. Glioblastoma multiforme: a review of where we have been and where we are going. Expert Opin Investig Drugs. 2009;18(8):1061-83.

2. Stupp R, Mason WP, van den Bent MJ, et al. Radiotherapy plus concomitant and adjuvant temozolomide for glioblastoma. N Engl J Med. 2005;352(10):987-96.

3. Curran Jr WJ, Scott CB, Horton J, et al. Recursive partitioning analysis of prognostic factors in three Radiation Therapy Oncology Group malignant glioma trials. J Natl Cancer Inst. 1993;85(9):704-10.

4. Kargiotis O, Rao JS, Kyritsis AP. Mechanisms of angiogenesis in gliomas. J Neurooncol. 2006;78(3):281-93.

5. Ferrara N. Vascular endothelial growth factor: basic science and clinical progress. Endocr Rev. 2004;25(4):581-611.

6. Hicklin DJ, Ellis LM. Role of the vascular endothelial growth factor pathway in tumor growth and angiogenesis. J Clin Oncol. 2005;23(5): 1011-127.

7. Gerber HP, Ferrara N. Pharmacology and pharmacodynamics of bevacizumab as monotherapy or in combination with cytotoxic therapy in preclinical studies. Cancer Res. 2005;65(3):671-80.

8. Yang JC, Haworth L, Sherry RM, et al. A randomized trial of bevacizumab, an anti-vascular endothelial growth factor antibody, for metastatic renal cancer. N Engl J Med. 2003;349 (5):427-34 .

9. Hurwitz H, Fehrenbacher L, Novotny W, et al. Bevacizumab plus irinotecan, fluorouracil, and leucovorin for metastatic colorectal cancer. N Engl J Med. 2004;350(23):2335-42.

10. Sandler A, Gray R, Perry MC, et al. Paclitaxel-carboplatin alone or with bevacizumab for non-small-cell lung cancer. N Engl J Med. 2006;355(24):2542-50.

11. Miller K, Wang M, Gralow J, et al. Paclitaxel plus bevacizumab versus paclitaxel alone for metastatic breast cancer. N Engl J Med. 2007;357(26):2666-76.

12. Mathieu V, De Néve N, Le Mercier M, et al. Combining bevacizumab with temozolomide increases the antitumor efficacy of temozolomide in a human glioblastoma orthotopic xenograft model. Neoplasia. 2008;10(12):1383-92.

13. Lee CG, Heijn M, di Tomaso E, et al. Anti-vascular endothelial growth factor treatment augments tumour radiation response under normoxic or hypoxic conditions. Cancer Res. 2000;60 (19):5565-70.

14. Vredenburgh JJ, Desjardins A, Herndon JE 2nd, et al: Phase II trial of bevacizumab and irinotecan in recurrent malignant glioma. Clin Cancer Res. 2007a;13(4):1253--9.

15. Vredenburgh JJ, Desjardins A, Herndon JE 2nd, et al. Bevacizumab plus irinotecan in recurrent glioblastoma multiforme. J Clin Oncol. 2007b;25(30):4722-9.

16. Norden AD, Young GS, Setayesh K, et al. Bevacizumab for recurrent malignant gliomas: efficacy, toxicity, and patterns of recurrence. Neurology. 2008;70(10):779-87.

17. Kreisl TN, Kim L, Moore K, et al. Phase II trial of single-agent bevacizumab followed by bevacizumab plus irinotecan at tumor progression in recurrent glioblastoma. J Clin Oncol. 2009;27(5): 740-5.

18. Friedman HS, Prados MD, Wen PY, et al. Bevacizumab alone and in combination with irinotecan in recurrent glioblastoma. J Clin Oncol. 2009;27(28):4733-40.

19. Cohen MH, Shen YL, Keegan P, Pazdur R. FDA drug approval summary: bevacizumab (Avastin) as treatment of recurrent glioblastoma multiforme. Oncologist. 2009;14(11):1131-8. 
20. Wen PY, Macdonald DR, Reardon DA, et al. Updated response assessment criteria for high-grade gliomas: Response assessment in neuro-oncology working group. J Clin Oncol. 2010;28(11): 1963-72.

21. Theeler BJ, Groves MD. High-grade gliomas. Curr Treat Options Neurol. 2011;13(4):386-99.

22. Yung WK, Albright RE, Olson J, et al. A phase II study of temozolomide vs. procarbazine in patients with glioblastoma multiforme at first relapse. Br J Cancer. 2000;83(5):588-93.

23. Wong ET, Gautam S, Malchow C, Lun M, Pan E, Brem S. Bevacizumab for recurrent glioblastoma multiforme: a metaanalysis. J Natl Compr Canc Netw. 2011;9(4):403-7.

24. Poulsen HS, Grunnet K, Sorensen M, et al. Bevacizumab plus irinotecan in the treatment patients with progressive recurrent malignant brain tumours. Acta Oncol. 2009;48(1):52-8.

25. Soffietti R, Ruda R, Trevisan E, et al. Phase II study of bevacizumab and nitrosourea in patients with recurrent malignant glioma: a multicenter Italian study. J Clin Oncol. 2009;27(suppl; abstr 2012).

26. Reardon DA, Desjardins A, Vredenburgh JJ, et al. Metronomic chemotherapy with daily, oral etoposide plus bevacizumab for recurrent malignant glioma: a phase II study. Br J Cancer. 2009; 101(12):1986-94.

27. Guiu S, Taillibert S, Chinot $\mathrm{O}$, et al. Bevacizumab/irinotecan. An active treatment for recurrent high grade gliomas: preliminary results of an ANOCEF Multicenter Study. Rev Neurol (Paris). 2008;164(6-7):588-94.

28. Gilbert MR, Wang M, Aldape K, et al. RTOG 0625: a phase II study of bevacizumab with irinotecan in recurrent glioblastoma (GBM). J Clin Oncol. 2009;27(suppl; abstr 2011).
29. Sathornsumetee S, Desjardins A, Vredenburgh JJ, et al. Phase II trial of bevacizumab and erlotinib in patients with recurrent malignant glioma. Neuro Oncol. 2010;12(12):1300-10.

30. Verhoeff JJ, Lavini C, van Linde ME, et al. Bevacizumab and doseintense temozolomide in recurrent high-grade glioma. Ann Oncol. 2010;21(8):1723-7.

31. Elandt $K$, Preusser $M$, Hassler $M$, et al. Treatment with bevacizumab and liposomal doxorubicin for recurrent highgrade gliomas (HGGs). J Clin Oncol. 2010;28(suppl; abstr e12522).

32. Calabrich A, Azevedo FC, Saad ED, et al. The feasibility and reallife effectiveness of the combination of chemotherapy (CT) and bevacizumab (Bev) in the treatment of high-grade gliomas (HGG) in a private community oncology center. J Clin Oncol. 2010;28(suppl; abstr e12552).

33. Gallardo Martin E, Areses Manrique MC, Anido Herranz U, et al. Efficacy, toxicity, and pattern of response and progression in patients with high-grade gliomas treated with bevacizumab and irinotecan: experience in one center. J Clin Oncol. 2010;28 (suppl; abstr e12553).

34. Vredenburgh JJ, Coughesy T, Samant M, et al. Corticosteroid use in patients with glioblastoma at first or second relapse treated with bevacizumab in the BRAIN study. Oncologist. 2010;15(12): 1329-34.

35. Henriksson R, Asklund T, Poulsen HS. Impact of therapy on quality of life, neurocognitive function and their correlates in glioblastoma multiforme: a review. J Neurooncol. 2011;104(3): 639-46. 